\title{
Characterisation of the Properties of a Negative Hydrogen Ion Beam by Several Beam Diagnostic Techniques
}

\author{
R. Maurizio ${ }^{1, \dagger}$, U. Fantz ${ }^{1}$, F. Bonomo ${ }^{1}$ and G. Serianni ${ }^{2}$ \\ ${ }^{1}$ Max-Planck-Institut für Plasmaphysik, Boltzmannstr. 2, 85748 Garching, \\ Germany \\ 2 Consorzio RFX, Euratom-ENEA association, Corso Stati Uniti 4, 35127 \\ Padova, Italy \\ E-mail: roberto.maurizio@epfl.ch
}

\begin{abstract}
.
The beam properties of the BATMAN negative ion source, which is the prototype of one module of the source for the ITER neutral beam injection system, are characterised by means of three diagnostic techniques: Beam Emission Spectroscopy (BES), the experimental calorimeter mini-STRIKE and a copper calorimeter. The main beam parameters - beam divergence, homogeneity and top-bottom asymmetries - are studied in different operational scenarios: with different magnetic filter field setups, source settings and with different gases (hydrogen or deuterium). Among all dependences, the influence of the magnetic field configuration on the beam and the evolution of the beam features during some conditioning days are investigated in detail. Data show that the stronger the filter field in the beam region, the higher the beam top-bottom asymmetry likely a $v \times B$ effect. During the conditioning of the source, such vertical beam asymmetry increases as well, suggesting an inhomogeneous $H^{-}$production at the first grid of the extraction system.
\end{abstract}

Keywords: Negative ion beam, beam diagnostics, Beam Emission Spectroscopy, Neutral Beam Injection, ITER

$\dagger$ Current address: École Polytechnique Fedérale de Lausanne (EPFL), Swiss Plasma Center (SPC), 1015 Lausanne, Switzerland 


\section{Introduction}

The ITER experiment will need external heating systems both to achieve ignition conditions and for current drive. One possibility is to use Neutral Beam Injectors $(\mathrm{NBI})$ in which negative ions of the same species of the plasma $\left(H^{-}\right.$or $\left.D^{-}\right)$are extracted from an ion source, accelerated to high energies, neutralized and finally injected into the plasma [1].

The RF driven cesiated negative hydrogen ion source is since 2007 the ITER NBI reference source; the development of this source aims to meet the ITERrelevant ion source parameters. The present research work is carried out in different test facilities [2, 3, 4, 5]. One of them is the test bed BATMAN (BAvarian Test MAchine for Negative ions), which is equipped with the prototype source; its main aim is the optimization of the RF source with respect to the extracted current density in hydrogen and deuterium, operating at low pressure $(0.3 \mathrm{~Pa})$ and electron-to-ion ratio $(<1)$, with a small extraction area $\left(<0.01 \mathrm{~m}^{2}\right)$ and short pulses $(<6 \mathrm{~s})$.

The paper provides a wide characterisation of the most important properties of BATMAN negative ion beam, namely beam divergence, vertical homogeneities and symmetries. This task is performed in several experimental conditions by means of three beam diagnostics: Beam Emission Spectroscopy, the miniSTRIKE calorimeter and a copper calorimeter.

An overview of the ion source and the beam diagnostic tools is given in section 2 ; beam divergence is also defined and a useful parameter for the characterisation of beam optics - the normalized perveance - is introduced. Section 3 contains the experimental results: particular emphasis is given to the effect of the magnetic field of the source on the beam and to the evolution of beam features with source conditioning. Finally operation with $H^{-}$and $D^{-}$ beams are compared.

\section{Beam diagnostic tools}

\subsection{Overview of the ion source}

The negative ion source of BATMAN is shown in Figure 1: a plasma is ignited in the RF driver - an alumina cylinder with a water-cooled RF coil wound on the outside of the cylinder that is connected to a $1 \mathrm{MHz}$ oscillator - and then expands into the source

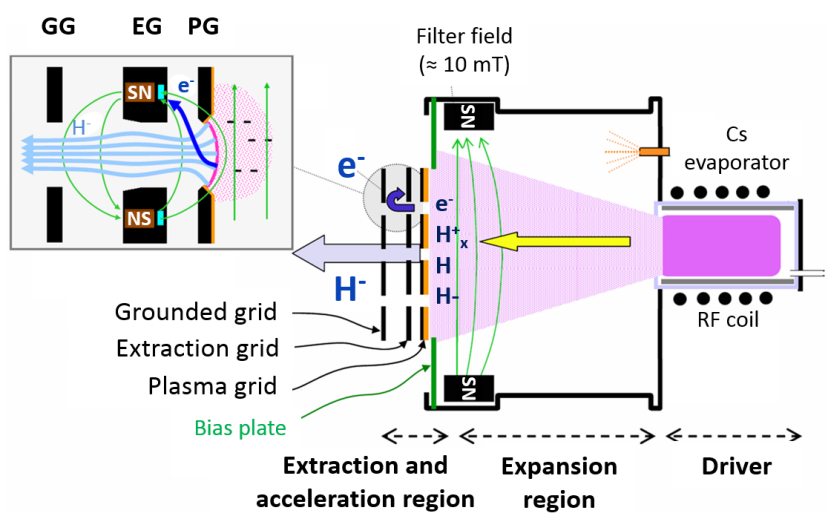

Figure 1. Schematic top view of the IPP RF source in BATMAN. The extraction and acceleration region is shown in the inlet; the magnets in the extraction grid are shown rotated by $90^{\circ}$.

body. A magnetic filter field - generated by internal or external magnets (shown in Figure 1) - is located in the expansion region; several magnetic setups can be explored [6]. The effect of the magnetic filter field is to reduce the electron flux to the region in front of the plasma grid (PG): because of their lower collisionality, hot electrons diffuse across the magnetic field more slowly than cold electrons, so that the fast electron flux will be reduced more than the one of slower electrons. Consequently, the electron density and temperature in front of the $\mathrm{PG}$ lower, leading to a reduction of the extracted electron current. Also, the destruction rate of negative ions (in the region in front of the PG) by collisions with electrons decreases since the efficiency of such process is proportional to the electron temperature.

Negative ions can be generated in two ways: in the plasma volume, by dissociative attachment of electrons on high vibrational states of hydrogen molecules [7], or on the first grid of the extraction system, which faces the plasma, by conversion of neutral or positive hydrogen ions to negative ions after their collision with the surface and the following capture of one or more electrons [8]. As regards the latter channel, often known as surface production, the probability of electron capture depends strongly on the work function of the surface, which can be lowered by covering it with Cs atoms. Cs is delivered by an oven on the top of the source back-plate: the nozzle is visible in Figure 1. The device has been recently upgraded: the Cs evaporation rate is controlled by the temperature of five different 


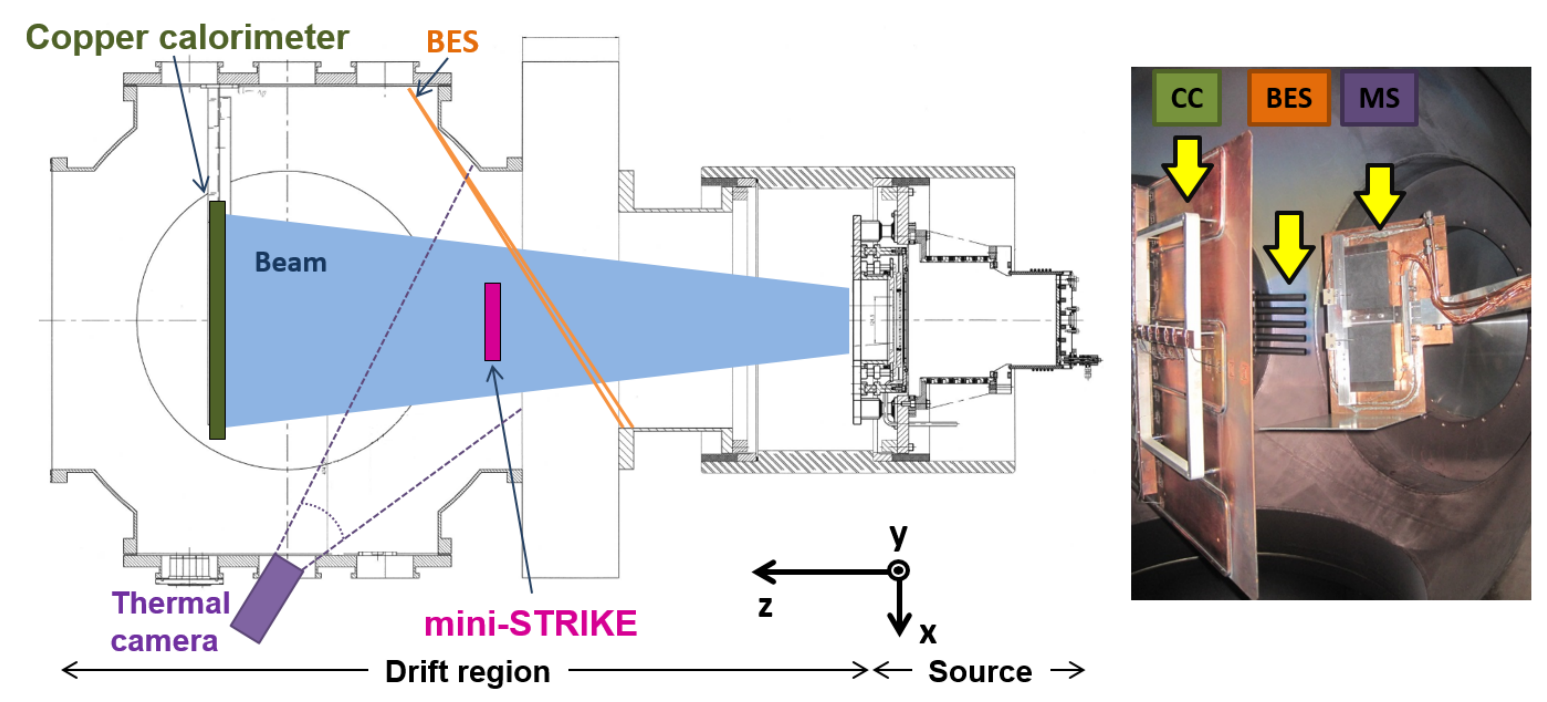

Figure 2. Top view of BATMAN drift region with beam diagnostics; on the right a photo taken inside the vacuum vessel is also presented.

locations of the oven, allowing high accuracy in the regulation of the evaporation rate, which is measured by a surface ionization detector (SID) located near the nozzle [9]. When Cs is present on the plasma grid, the surface production by atoms impacting the grid and creating negative ions dominates over the generation of negative ions in the plasma volume.

The negative ions are extracted from the source and accelerated up to $25 \mathrm{kV}$ by a system of three multiaperture grids at different potentials: the plasma grid (PG), extraction grid (EG) and grounded grid (GG) (Figure 1). The current geometry of the grids is known as LAG (Large Area Grid): each grid is made of two identical halves with 63 apertures each arranged in six rows, with an aperture diameter of $0.8 \mathrm{~cm}$ and a total extraction area of $63 \mathrm{~cm}^{2}$ [2]. As a result, the beam consists of 126 beamlets at the exit of the last grid (GG). Both grid halves are tilted by $1.75^{\circ}$ with respect to the vertical direction for beam focusing; this feature does not affect BES measurements, since the line of sight of a BES telescope is horizontal. Although an accurate mounting of the grid halves by using pins at different positions (precision of $20 \mu \mathrm{m}$ ), a small horizontal misalignment between them could exist: indeed during each beam pulse the grid expands due to the higher temperature. In this eventuality which cannot be excluded since no dedicated studies have been performed so far - the central BES telescope, located on the equatorial plane, would see the overlap of beamlets from both halves, resulting into a slight overestimation of the local beam divergence (see next section). The first gap - between the PG and the EG - is $3.5 \mathrm{~mm}$ large with a typical voltage up to $10 \mathrm{kV}$; the second gap - between EG and GG - is $8 \mathrm{~mm}$ large with a typical voltage of $15 \mathrm{kV}$.

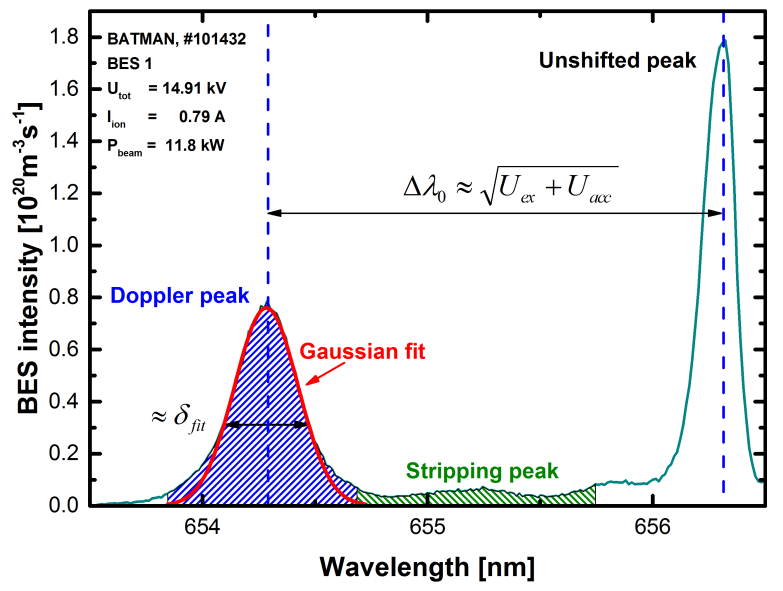

Figure 3. Typical BES spectrum, with three lines visible: the $H_{\alpha}$ unshifted line (on the right), the $H_{\alpha}$ Doppler- shifted line (on the left) and in the center a broad stripping peak.

Once extracted and accelerated the negative ions enter the drift region (Figure 2). Three beam diagnostics are available at BATMAN: they consist of a vertical array of five telescopes for spectroscopy (Beam Emission Spectroscopy, BES), a diagnostic calorimeter made up of two Carbon-Fiber-Composite tiles (named mini-STRIKE) and finally a copper calorimeter located as shown in Figure 2.

\section{2. $B E S$}

Beam emission spectroscopy, known also as Dopplershifted $H_{\alpha}$ emission spectroscopy, is generally used to estimate the divergence and the stripping losses of the 
beam; this is performed with the evaluation of the light-spectrum produced by the interaction between the beam and the background gas in the drift region $[10,11,12]$.

The emitted light is collected along five horizontal lines-of-sight (LOS), as shown in Figure 2; each telescope, labeled 5 to 1 from the highest to the lowest, has a mean angle, with respect to the beam axis, of $\theta_{0}=52^{\circ}$ and the vertical distance between two LOS is $40 \mathrm{~mm}$. The average distance between the telescope LOS intercepting the beam and the grounded grid is about $0.6 \mathrm{~m}$. At that location the 126 beamlets that form the beam are already superimposed due to their divergence. Each BES telescope sees the overlay of many beamlets and thus it can only measure line-ofsight averaged properties of the beam along one specific horizontal layer; the "beam divergence" given by a BES telescope must be intended as an average between the divergences of all the beamlets within the line-of-sight of that telescope.

The light collected by each telescope is then analyzed by a spectrometer in the wavelength range around the $H_{\alpha}$ line (the first line of the Balmer series for hydrogen); a typical BES spectrum, once calibrated to photon intensity, is shown in Figure 3. The beam power indicated in the figure is obtained by multiplying the electrically measured ion current $I_{i o n}$ with the sum of the extraction and acceleration voltage $U_{\text {tot }}$; such value is an overestimation of the actual beam power, since usually only $80 \%$ of $I_{\text {ion }}$ is found on the calorimeter at the end of the vacuum vessel, mainly because of losses onto the grounded grid [2]. A direct measurement of the beam power hitting the copper calorimeter was not possible for the shots here analyzed: indeed when mini-STRIKE is mounted, it intercepts the beam (see Figure 2).

In a typical BES spectrum three peaks can be detected: the unshifted $H_{\alpha}$ peak (at about $656.3 \mathrm{~nm}$ ), the Doppler-shifted peak (at about $654.5 \mathrm{~nm}$ ) and finally, between them, the stripping peak. The first is the line emission caused by the dissociation and excitation of the background gas (the same gas of the plasma, usually $H_{2}$, at a typical tank pressure of [1 to 5] $10^{-4}$ mbar) induced by collisions with highenergy neutrals:

$H_{f}^{0}+H_{2} \rightarrow H_{f}^{0}+H_{s}^{0}(n=3)+H_{s}^{0}(n=3)$

The indices $s$ and $f$ stand respectively for slow (background) and fast (beam) particles; the $H_{s}^{0}$ created are in the excited state $n=3$ and spontaneously decade to the lower state $n=2$. This leads to the emission of photons with wavelength $\lambda_{0}=$ $656.3 \mathrm{~nm}$ (first line of the Balmer series, $H_{\alpha}$ ). The second peak, the Doppler-shifted peak, is generated when fully-accelerated beam particles $H_{f}^{-}$undergo the electron detachment process (Equation 2) and when fast neutrals $H_{f}^{0}$, created by collisions with the background gas, get excited after colliding again with background atoms (Equation 3) [13, 14] :

$H_{f}^{-}+H_{2} \rightarrow H_{f}^{0}(n=3)+H_{2}+e^{-}$

$H_{f}^{0}+H_{2} \rightarrow H_{f}^{0}(n=3)+H_{2}$

Collisions of positive ions (generated by ionization along the beam path) with the background gas can be neglected here due to the small relative velocity of the particles within the beam [15]. The fast neutral $H_{f}^{0}(n=3)$ can thus decay from the excited state $n=3$ to $n=2$, emitting a photon at $\lambda_{0}=656.3$ $\mathrm{nm}$. However, due to the motion of the neutral with respect to the laboratory frame, the emitted light is Doppler-shifted of $\Delta \lambda$ :

$\Delta \lambda=-\lambda_{0} \cos \theta v / c$

where $\lambda_{0}$ is the proper wavelength (in the particle frame), $\theta$ is the viewing angle of the telescope with respect to the beam axis, $v$ is the velocity of the particle and $c$ the speed of light in vacuum.

The entity of the Doppler shift is proportional to the total energy of the particle $(\Delta \lambda \propto v \propto$ $\sqrt{U_{e x}+U_{a c c}}$, where the extraction voltage $\left(U_{e x}\right)$ is the voltage drop between the $\mathrm{PG}$ and $\mathrm{EG}$, the acceleration voltage $\left(U_{a c c}\right)$ between the EG and the GG). Moreover, line broadening $\delta(\Delta \lambda)$ will occur as a result of a distribution of $\theta$ (natural divergence of the beam $\epsilon=\delta \theta$, measured in degrees and thoroughly described in section 2.5) and a distribution of the beam particles energy around the mean value $e \cdot\left(U_{e x}+U_{a c c}\right)$. Finally, part of the negative ions are neutralized between the $P G$ and the $G G$ via the stripping reactions:

$H_{f}^{-}+H_{2} \rightarrow H_{f}^{0}+H_{2}+e^{-}$

$H_{f}^{-}+H_{2} \rightarrow H_{f}^{+}+H_{2}+2 e^{-}$

The fast neutrals produced (reaction 5) are then excited by collisions with the background gas of the tank to the level $n=3$ and subsequently relax to $n=2$. The light emitted is partially Doppler-shifted, since the stripped particles do not have the full beam energy $\left(U_{e x}+U_{a c c}\right)$, but usually only the energy of the extraction gap. The typical extraction and acceleration voltages are $5 \mathrm{kV}$ and $15 \mathrm{kV}$ respectively: thus the typical energy for a stripped ion is around $5 \mathrm{keV}$, for a fully-accelerated ion is $20 \mathrm{kV}$.

The line-of-sight averaged beam divergence $\epsilon$ can be measured starting with an estimate of the $(1 / e)-$ width (e-folding width) of the Doppler-shifted line given by a Gaussian interpolation, $\delta(\Delta \lambda)_{f i t}[12]$. Then some corrections are applied in order to consider undesirable phenomena that broaden the line width $[12,16]$; the corrected quantity, $\delta(\Delta \lambda)_{a p p}$ "apparent 

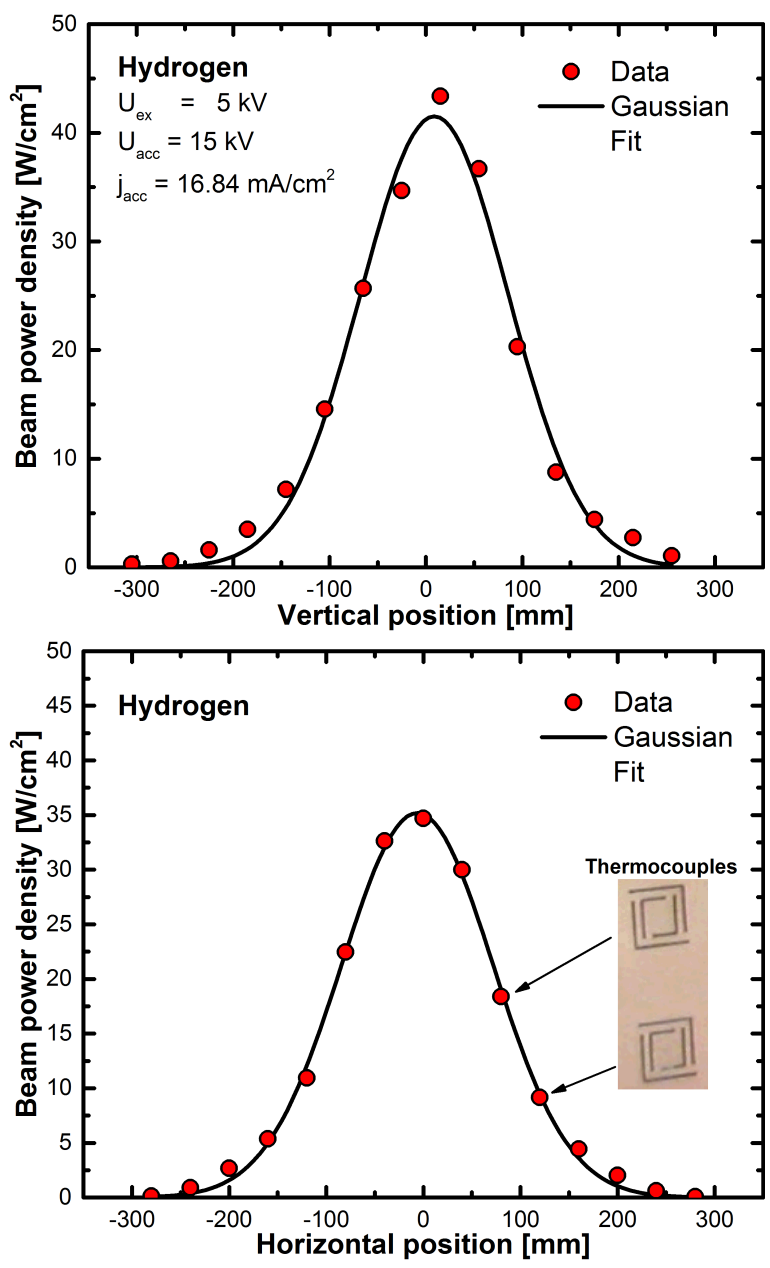

Figure 4. Typical beam profiles, along the vertical and horizontal direction, on the copper calorimeter, measured with thermocouples. The inset shows two thermally-isolated areas of the calorimeter on the beam facing side: behind each area a thermocouple is installed.

width", is directly correlated to the beam divergence $\epsilon$ :

$\delta(\Delta \lambda)_{a p p}=\Delta \lambda_{0} \cdot \tan \theta_{0} \cdot \epsilon$

where $\Delta \lambda_{0}$ is the mean Doppler shift of the $H_{\alpha}$ line.

\section{3. $\operatorname{mini}-S T R I K E$}

mini-STRIKE is a small-scale version of the STRIKE calorimeter (Short-Time Retractable Instrumented Kalorimeter Experiment), which will be one of the main diagnostics of the negative ion source SPIDER of the PRIMA test facility [17]. mini-STRIKE consists of 2 CFC tiles $\left(120 \times 90 \times 20 \mathrm{~mm}^{3}\right)$ which are observed in the rear side (the side not exposed directly to the beam, to avoid light from overheated carbon dust on the tile surface) by a thermal camera. In the last few years its diagnostic capabilities have been widely assessed in test facilities equipped with a negative ion source:

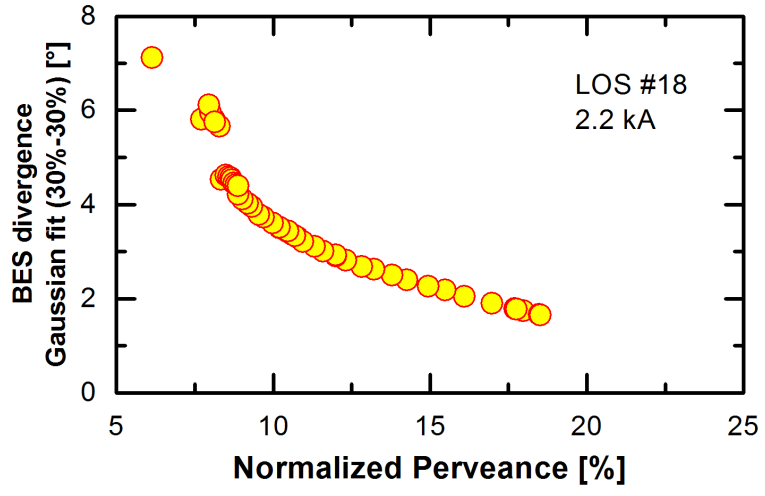

Figure 5. Line-of-sight averaged beam divergence as a function of normalized perveance, for one of the vertical BES of ELISE.

BATMAN test facility (IPP-Garching, Germany, [18] [19]) and the test stand of the negative-ion-driven neutral beams for the LHD stellarator (NIFS-Toki, Japan, [20]).

The experimental setup of mini-STRIKE at BATMAN is visible in Figure 2; a more detailed description is given in [18]. The CFC tiles are vertically arranged and housed in a dedicated frame which is connected to a supporting arm attached to the large BATMAN vacuum flange: the tiles are exposed to the beam at a position $1 \mathrm{~m}$ downstream with respect to the extraction system, in order to avoid any carbon contamination of the source.

At that location the footprint of the beamlets are calculated to superimpose on the footprints from neighboring beamlets due to their divergence, leading to a wide "bell-shaped" beam profile. To re-create a beamlet pattern on mini-STRIKE, an actively-cooled copper mask with several holes is positioned just in front of the tiles: the beam is intercepted by the mask, so that the beam passing through each aperture produces a footprint on the tile similar to the one expected from a single beamlet of the SPIDER negative ion beam on the STRIKE diagnostic. An automatic analysis procedure has been set up to analyze the videos recorded by the thermal camera. Each thermal video is decomposed in 500 frames (FPF images), which constitute the input of the code: the algorithm performs a complete parametrization of the beam as it is seen in the rear side of the tiles, and a list of significant "beam parameters" is provided as output. In the following, the features of the temperature 2Dprofile on the masked tiles will be improperly referred to as the "beam pattern"; a thorough description of the code can be found in [19].

The function which interpolates the beam behind the tiles is the product of a parabola in the horizontal direction (8) and a skewed-Gaussian in the vertical (9), 
namely a Gaussian with non-zero skewness:

$p(x)=a x^{2}+b x+c$

$g(y)=\exp \left[-\left(\frac{y-y_{0}}{4 \omega}\right)^{2}\right]\left[1+\operatorname{erf}\left(\alpha \frac{y-y_{0}}{\sqrt{2} \omega}\right)\right]$

$h(x, y)=p(x) \cdot g(y)$

where $a, b, c, \omega, y_{0}, \alpha$ are free parameters, while erf is the error function.

\subsection{Copper calorimeter}

A copper calorimeter intercepts the beam at the end of the vacuum vessel, approximately $1.7 \mathrm{~m}$ downstream of the grounded grid (see Figure 2). It consists of a water-cooled copper panel $\left(60 \times 60 \mathrm{~cm}^{2}\right)$ with specially-designed thermally-isolated areas where 29 thermocouples are embedded. They are arranged as a cross and the distance between two consecutive thermocouples is $4 \mathrm{~cm}$; the center of the cross is shifted $3 \mathrm{~cm}$ downwards with respect to the nominal centre-line of BATMAN because of a technical problem during the mouting [21]. During the beam phase, the temperature time trace of each thermocouple is recorded and then the $\Delta T$ between the start and the end of the beam is computed [2]; since each thermocouple is thermally isolated from the rest of the calorimeter, the local beam power density can be calculated from the measured $\Delta T$.

Typical vertical and horizontal power density profiles measured by the calorimeter are shown in Figures 4: evaluation of the profiles, performed up to now with a simple Gaussian fitting, leads to an estimate of both the beam divergence at the calorimeter position and the total beam power impinging on the plate [22].

\subsection{Source parameters, divergence and beam profile}

One of the most important properties of a charged beam is the divergence of the beamlets making up the beam. This quantity basically describes the angular spread of the particle trajectories around the beamlet axis: a good beamlet is characterised by a low divergence, a bad beamlet by a high divergence.

If we consider a beamlet which exits from a circular aperture of the grounded grid and drifts in the vacuum vessel, its width $\sigma(z)$ will increase with the distance $z$ from the grounded grid: indeed, due to space-charge repulsion, the particle trajectories will deviate from the beamlet axis. At a certain location downstream the GG, the positive space charge of the background compensates for the negative space charge of the beamlet and its divergence stops increasing; in the case of the MANITU test facility, for instance, the full space charge compensation sets in at a distance of $12 \mathrm{~cm}$ from the GG [23]. For BATMAN, which works with a typical tank pressure much higher than in MANITU, recent studies have shown that the beam charge is fully compensated a few centimeters downstream of the last grid [24].

The distribution of the angle between each trajectory and the beamlet axis is generally assumed to be Gaussian, centered in zero; the beamlet divergence $\delta$ is by definition the e-folding half-width of such Gaussian, $\sqrt{2} \sigma$ ( $\sigma$ is the standard deviation). The divergence of a beamlet depends on the current in the beamlet, on the voltages applied to the extraction and acceleration grids and on their geometry. The grids constitute indeed a system of electrostatic lenses [25], and a strong analogy between charged and light beams is usually adopted: as a light beam can be focused or de-focused by the lenses, similarly a charged beam can be focused or de-focused by the electric or magnetic fields between the apertures of the grids.

A useful parameter in the characterisation of the beam optics is the perveance, usually defined as the ratio of the ion current $I$ to the three-halves power of the applied voltage $U[26]$ :

$\Pi=I / U^{3 / 2}$

The maximum possible extracted current $I_{\max }$ for a radially symmetric ion beamlet is limited by the space charge of the beamlet (Child-Langmuir Law); the maximum perveance $\Pi_{0}$ can thereby be computed, and the normalized perveance of the beam is by definition the ratio $\Pi / \Pi_{0}$. Also the normalized perveance depends on the square root of the ion mass $\Pi / \Pi_{0} \propto$ $\sqrt{m}[26]$.

As already mentioned, the grid system of BATMAN test facility consists of three grids; since two voltages are present in the gaps between the grids, the extraction voltage $U_{e x}$ and acceleration voltage $U_{a c c}$, also two normalized perveances can be defined. The reference parameter which is here taken for describing the beam optics condition is the normalized perveance obtained from the extracted current density $j_{e x}$ and the extraction voltage.

The experimental dependence of the beam divergence on the normalized perveance, is found to be parabolic (Figure 5); the divergence shown is estimated using a vertical BES line-of-sight of ELISE test facility [3]. The perveance was varied by changing the extraction voltage. The left branch of the parabola, where the divergence decreases with increasing perveance, is known as under-perveance region; the right branch, seldom explored in BATMAN (since it corresponds to very high beam currents), is known as over-perveance region. The broad minimum of the divergence, corresponding to the optimum of the perveance, is also visible. 


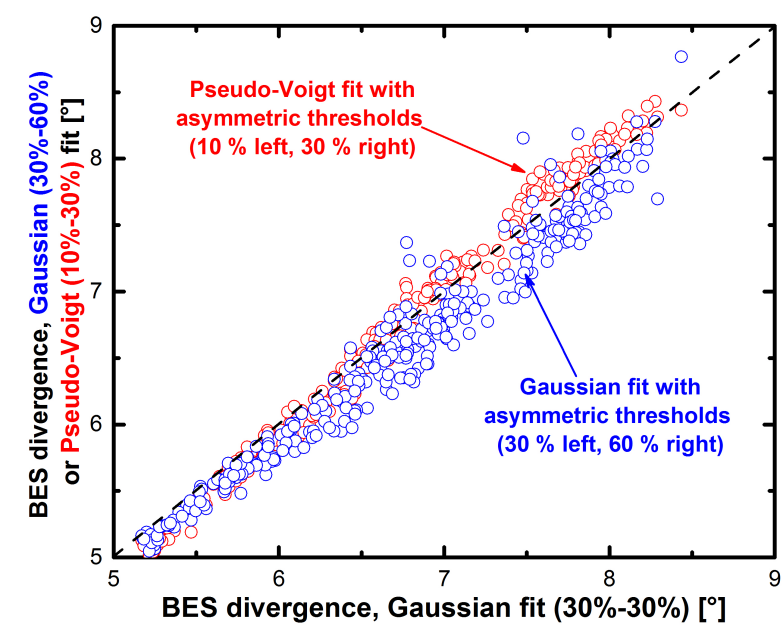

Figure 6. BES divergence (LOS 1 to 5) from Pseudo-Voigt fit (red points) and Gaussian fit with asymmetric thresholds (blue points), plotted against the divergence from standard evaluation.

\section{Results}

\subsection{Strength and weaknesses of the methods}

Different fitting techniques of the Doppler-shifted peak have been explored, in order to understand whether the beam divergence given by Beam Emission Spectroscopy depends on the choice of the fitting function. A precise approximation of the Dopplershifted line is crucial to get a stable estimate of the beam divergence: both the background fluctuations and possible contaminations from the broad stripping peak must be taken into account when the fitting function is chosen [27].

This can be done by selecting carefully the fitting range: a specific threshold is applied on the left and right side of the peak so that solely the portion of the peak exceeding a given percentage of its maximum, e.g $30 \%$, is interpolated. In some experimental situations the stripping peak contaminates evidently the righthalf of the Doppler line and the $30 \%$ threshold can be not enough: such contamination can be avoided by increasing the right-side threshold to $60 \%$.

Three different fitting alternatives have been explored: (1) a Gaussian fit with symmetric thresholds (30\% on the left and on the right), (2) a Gaussian fit with asymmetric thresholds $(30 \%$ on the left and $60 \%$ on the right) and finally (3) a Pseudo-Voigt interpolation with asymmetric thresholds $(10 \%$ on the left and $30 \%$ on the right). The first case is the one adopted in the standard evaluation used at BATMAN.

A Pseudo-Voigt $V(\lambda)$ is the linear combination between a Lorentzian $L(\lambda)$ and a Gaussian $G(\lambda)$ :

$V(\lambda)=\eta \cdot L\left(\lambda ; \lambda_{0}, \omega\right)+\beta \cdot G\left(\lambda ; \lambda_{0}, \omega\right)$
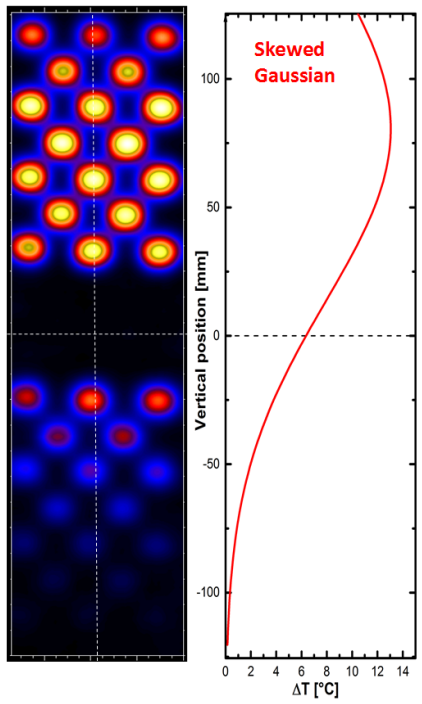

Figure 7. Typical thermal pattern on the rear side of miniSTRIKE; the vertical section of the beam fitting function is also shown.

where

$L(\lambda)=\frac{1}{\pi \omega}\left[1+\left(\frac{\lambda-\lambda_{0}}{\omega}\right)^{2}\right]^{-1}$

and

$G(\lambda)=\sqrt{\frac{\ln 2}{\pi}} \frac{1}{\omega} \exp \left\{-\ln 2\left(\frac{\lambda-\lambda_{0}}{\omega}\right)^{2}\right\}$

while $\omega, \lambda_{0}, \eta$ and $\beta$ are free parameters. Typical values for the Lorentzian and Gaussian weights, $\eta$ and $\beta$, are 0.6 and 0.4 respectively.

In Figure 6 the beam divergence given by the five BES telescopes using method (2) and (3) is plotted against the divergence from method (1); data refer to a typical day of $\mathrm{Cs}$ conditioning in hydrogen. The difference in divergence between the three methods is very small along the entire range $[5 \text { to } 8]^{\circ}$; the same can be shown as regards the intensity of the Doppler line, referred to as "BES intensity" and measured in $m^{-3} s^{-1}$. The three methods are therefore equivalent within the error uncertainties: the estimate of beam divergence does not depend on the choice of the line fitting function.

The Gaussian with symmetric thresholds (method 1) has been chosen as the interpolating function for the $H_{\alpha}$ Doppler-shifted peak, in agreement with the standard evaluation used at BATMAN.

The strength and weaknesses of mini-STRIKE can be deduced by observing a typical thermal pattern on the rear side of the tiles (Figure 7). With the current design of the copper mask which filters the beam in front of the calorimeter, the spatial resolution in the vertical direction ( $8 / 7$ holes alternating) is far better than in the horizontal direction ( 2 / 


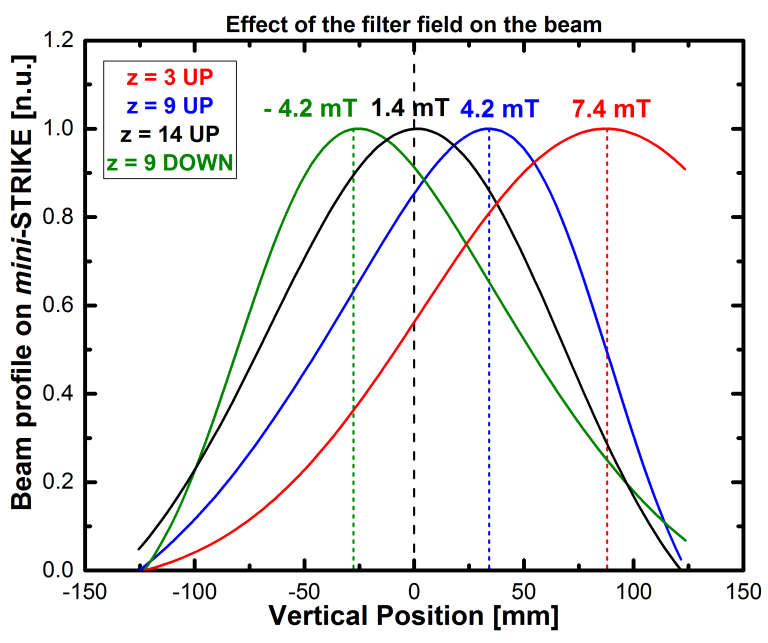

Figure 8. Beam vertical profiles at mini-STRIKE for different magnetic setups: the strength of the magnetic field at the grounded grid location is also indicated.

3 holes alternating): for now mini-STRIKE is a useful tool to investigate the beam vertical profile but not the horizontal. This is not an issue for the characterisation of BATMAN beam, since no relevant beam asymmetries are expected in the horizontal direction; on the contrary, top-bottom beam asymmetries can in principle appear as a result of the $v \times B$ interaction of the ions with the (horizontal) filter field.

As regards the copper calorimeter, its diagnostic capabilities are the same in the vertical and horizontal direction since the distance between two consecutive thermocouples on the plate is always $4 \mathrm{~cm}$. The copper calorimeter ability of characterising vertical and horizontal asymmetries is worse than mini-STRIKE: this is because it has a lower spatial resolution and is more distant from the grounded grid $(1.7 \mathrm{~m}$, miniSTRIKE is at $1 \mathrm{~m}$ ). Indeed, since the beam is divergent, its vertical and horizontal profiles smooth as the beam drifts and all asymmetries are expected to naturally reduce with the distance from the last grid.

\subsection{Effect of the filter field on beam profile}

The beam vertical properties are investigated looking at the vertical section of the thermal pattern on the rear side on mini-STRIKE, at the vertical array of $\Delta T$ given by the thermocouples on the copper calorimeter and finally at the distribution of the Doppler-shifted $H_{\alpha}$ light intensity collected by the five BES telescopes.

The beam vertical profile is usually expected to depend primarily on two factors:

(i) the ion trajectory inclination, correlated to the $v \times B$ interaction between the beam particles and the (horizontal) filter field in the extraction,

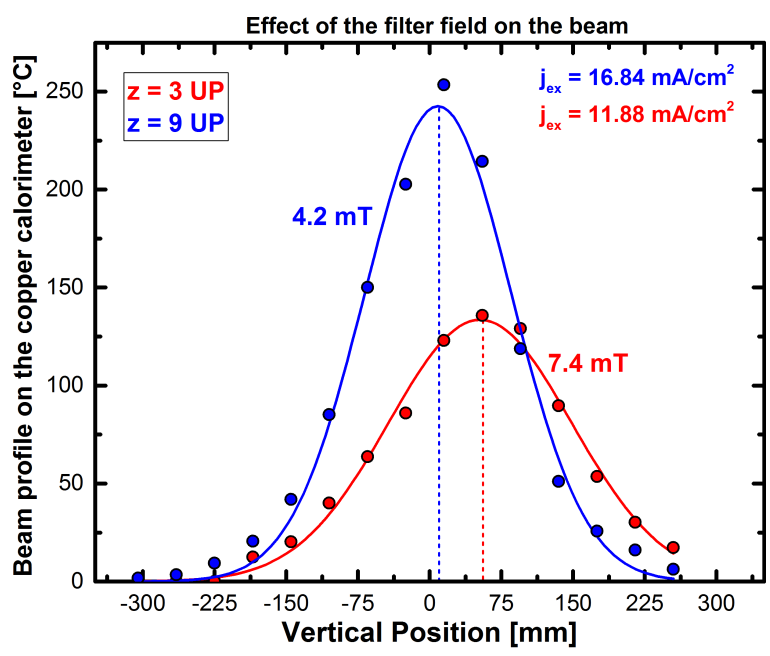

Figure 9. Beam vertical profiles at the copper calorimeter for different magnetic setups: the extracted current $j_{e x}$ changes because of different conditioning of the source. The strength of the magnetic field at the grounded grid location is also indicated.

acceleration and drift regions, i.e. the beam deflection;

(ii) the vertical distribution of the extracted current at the plasma grid, which is influenced by the Cs coverage of the grid surface and the plasma drift caused by the filter field [6].

The influence of the vertical non-uniformity of the plasma on the negative ion production - point (ii) depends on the relevance of the positive ions in the surface conversion process. When the atomic hydrogen flux towards the plasma grid is sufficiently strong, the emission of negative ions from the surface into the plasma, which is space charge limited, is enhanced by the local density of positive ions [28]. This condition is usually true for the shots here analyzed.

The dependence (ii) will be thoroughly discussed in section 3.3; effect (i) is due to the unavoidable penetration of the filter field from the source volume into the grids and drift regions.

In the extraction and acceleration region the average ion velocity is lower than in the drift region and the filter field is stronger: it is reasonable to conclude that the region between the grids influences the overall ion deflection more that the drift region. Several magnetic setups are available in BATMAN, each labeled with the distance $z$ of the magnetic field maximum from the plasma grid (source side) and with the direction of the plasma drift in front of such grid (up or down): a top-bottom plasma asymmetry in front of the PG has indeed been experimentally observed $[6,15]$. For instance, with the $\mathrm{z}=3 \mathrm{UP}$ setup the field strength is maximum $3 \mathrm{~cm}$ from the $\mathrm{PG}$ and the plasma is drifted upwards in front of the grid; by changing to $\mathrm{z}=9$ and $\mathrm{z}=14$ setups, the field strength in the 


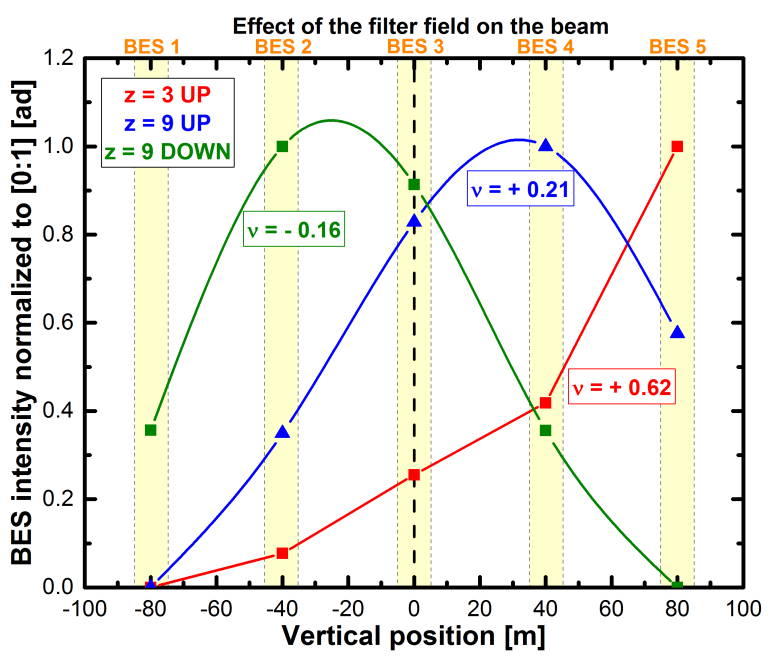

Figure 10. BES intensity profiles (normalized to [0:1]) for three magnetic setups: each one is labeled with its asymmetry factor.

source increases but it weakens in the grid and drift regions. Furthermore, by inverting the magnets also DOWN configuration can be explored [29, 30].

The experimental results are quite clear (Figure 8): at fixed source settings, the stronger the filter field in the drift region, the higher the deviation of the beam from the horizontal. In the plot each beam profile is labeled with the field strength at the grounded grid location. Moreover, the beam profiles before and after the inversion of field polarity, $\mathrm{z}=9 \mathrm{UP}$ and $\mathrm{z}=9$ DOWN, are symmetric with respect to the zero of BATMAN, which is the vertical position of the horizontal plane of symmetry of the source: such symmetry implies that the vertical location of the beam center if no filter field were applied essentially coincides with the zero of BATMAN.

Similar conclusions can be drawn by observing the vertical profile provided by the copper calorimeter (Figure 9) or the "spectroscopic" beam profile (Figure 10). The latter is obtained by considering the vertical distribution of BES intensity; such quantity is indeed, in very first approximation, proportional to the beam current visible by BES telescope. To measure the level of beam top-bottom asymmetry with spectroscopy, an asymmetry factor $\nu$ has been defined as a proper combination of the five BES intensities $I_{1} \ldots I_{5}$ :

$\nu:=\frac{\left(I_{4}+I_{5}+0.5 \cdot I_{3}\right)-\left(I_{1}+I_{2}+0.5 \cdot I_{3}\right)}{\left(I_{1}+I_{2}+I_{3}+I_{4}+I_{5}\right)}$

Following this definition, a perfectly symmetric beam has an asymmetry factor of zero; $\nu$ is positive for a shifted-up beam, negative for a shifted-down beam and the greater its absolute value, the higher the beam inhomogeneity. In summary, the three diagnostics agree well within their results: the common outcome of data analysis is that beam asymmetry and

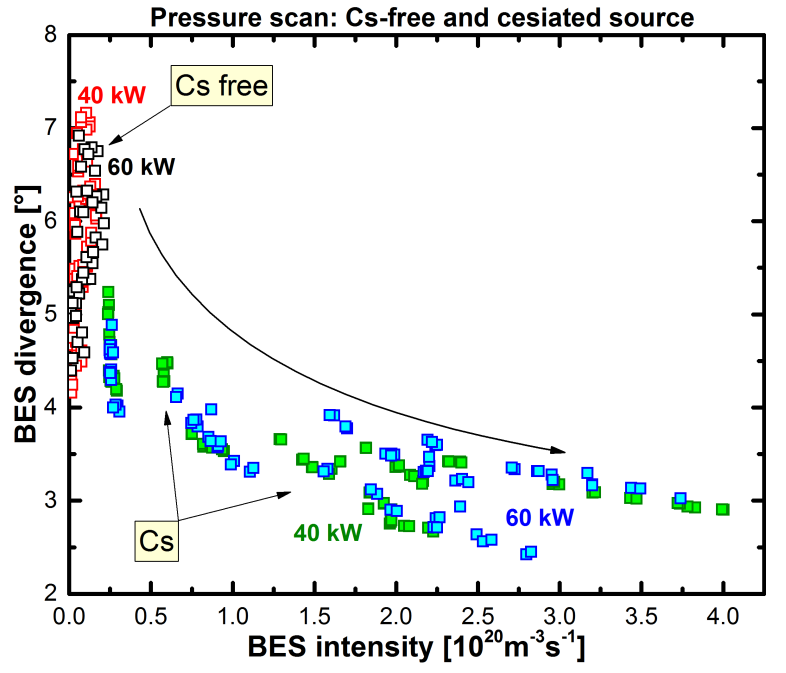

Figure 11. BES divergence for telescopes 1 to 5 , as a function of the correspondent BES intensity: data refer to some pressure scans performed at different RF powers (indicated) with $\mathrm{z}=3$ UP.

homogeneity change with the position of the magnets and thus with the strength of the filter field in the beam region.

\subsection{Divergence and source conditioning}

Source conditioning consists in the repetition of the same beam pulse (fixed settings) while Cs is introduced in the source continuously, during both the vacuum phase and the plasma pulses; the PG surface work function gradually lowers as a result of Cs coverage, leading to a higher amount of extracted current $j_{e x}$ and beam normalized perveance. This affects deeply the beam optics, as mentioned in section 2.5.

In Figure 11 the BES intensity from each telescope is plotted against the correspondent divergence. Such figure reproduces the parabolic relationship between beam divergence and normalized perveance (Figure 5) since the BES intensity and the normalized perveance $\Pi / \Pi_{0}$ are roughly proportional: indeed the BES intensity is in the very first approximation proportional to the local current density, which is linearly proportional to the local perveance. Furthermore, the key beam features - current and divergence - change drastically passing from a Cs-free source (empty symbols) to a cesiated one (full symbols). A detailed analysis of some Cs-conditioning days in hydrogen, at $\mathrm{z}=3 \mathrm{UP}$, evidenced two interesting and quite unexpected aspects: (1) a beam top-bottom asymmetry increasing with conditioning and (2) a non homogeneous behavior of the beam as regards the transition from under-perveance to over-perveance. The aspect (1) is seen by the calorimeters as a drift upwards of the beam profile (Figures 12), by the 

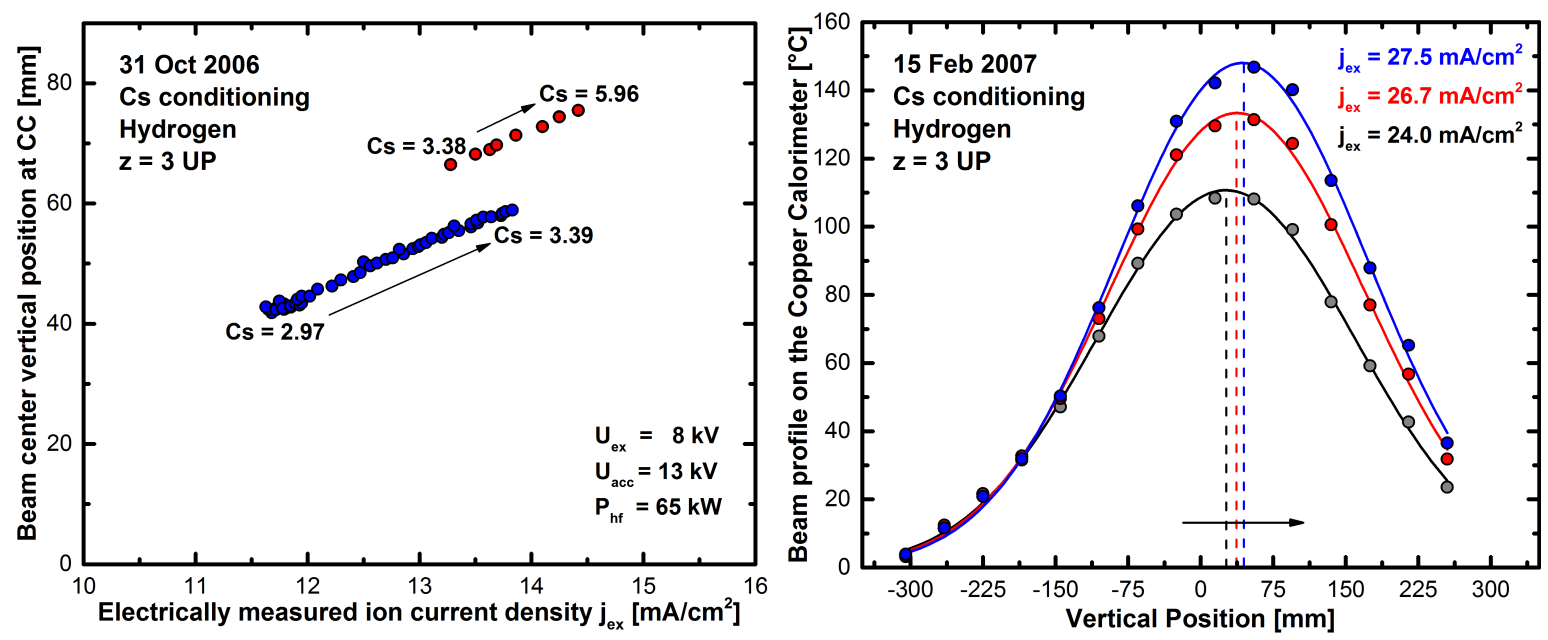

Figure 12. (Left) Beam center vertical position from the copper calorimeter, during a conditioning day: the blue and red symbols are separated by a series of beam pulses not considered because one of the source settings, the current collected at the PG (known as bias current, $I_{\text {bias }}$ ), was modified . (Right) Beam vertical profiles from the copper calorimeter; the increase of the area under the curve reflects the increase in the amount of current, due to different source conditioning.
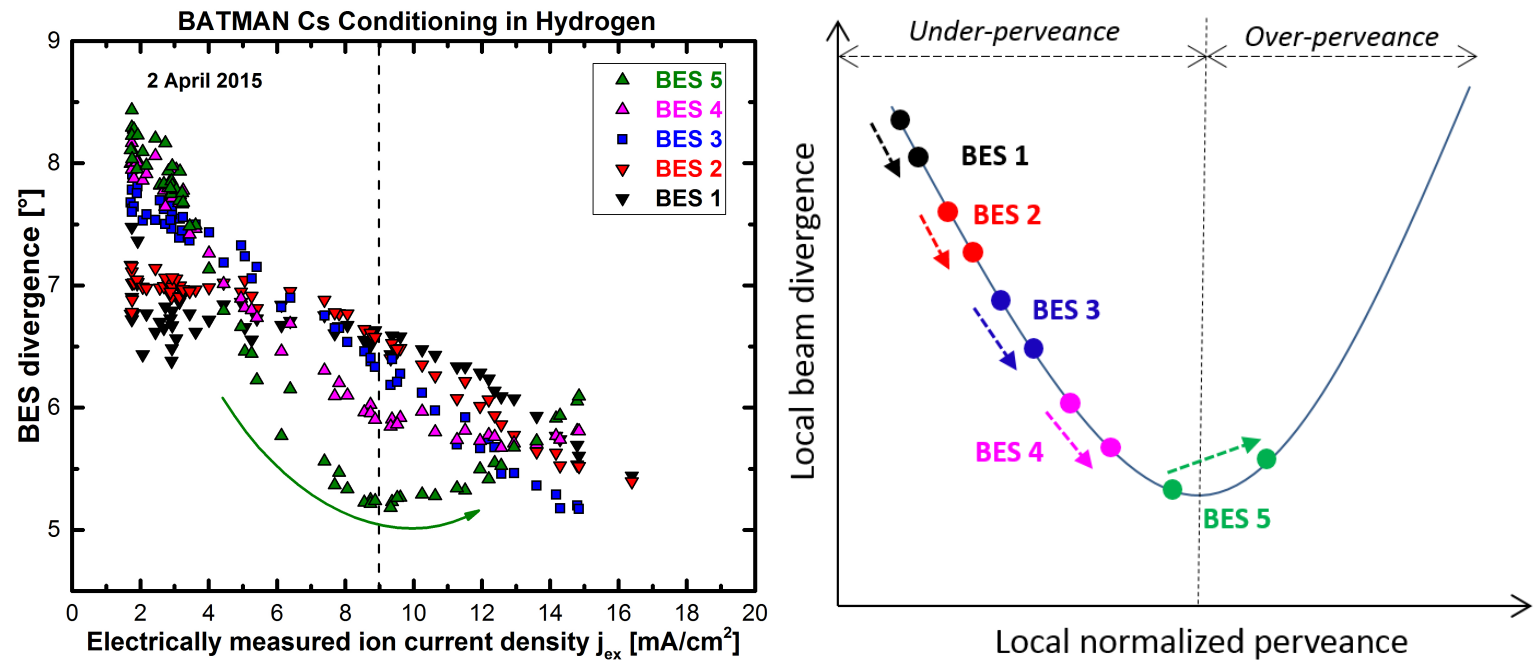

Figure 13. (Left) BES divergence as a function of the electrically measured current density: for telescope \#5 the transition from under-perveance to over-perveance is visible. (Right) Schematic evolution of the five BES divergences during a conditioning day.

spectroscopy as an increase of the asymmetry factor $\nu$.

An inhomogeneous Cs coverage of the PG would easily explain effect (1). From a theoretical point of view, since the Cs-oven nozzle is located at the top of the rear wall of the source, during the vacuum phase the Cs atoms move toward the PG and cover mainly the upper half, creating a relevant vertical asymmetry of the surface work function. However, this asymmetry should be strongly reduced if a plasma is ignited: the plasma is expected to erode the Cs from the surface, ionize the bulk of the Cs atoms and thus redistribute them on all the surfaces with which the plasma interacts (as the lateral walls and the lower half of the PG) [31]. The experimental evidences, however, suggest that at $\mathrm{z}=3 \mathrm{UP}$ and a RF power of 60 to 65 $\mathrm{kW}$ the erosion and redistribution effect of the plasma on the PG is not strong enough: a residual top-bottom asymmetry remains and it increases with time.

It is worth to notice that measurements from the source-side - namely laser absorption - show that the Cs density in front of the PG is not homogeneous: during a recent conditioning day (April 2015) a factor 3 to 4 was found to exist between the line-of-sight averaged density of neutral Cs in the plasma in front of the top and in front of the bottom of the plasma grid [32]. During the Cs conditioning phase of the ion source a dependence of the source performance on the measured Cs density in front of the plasma grid on a short timescale (one day) occurs, but due 


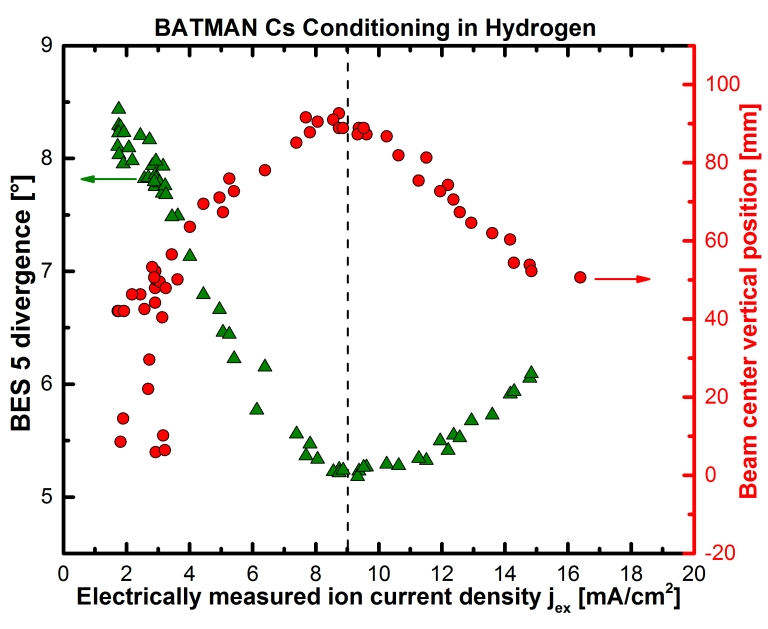

Figure 14. BES 5 divergence and beam center vertical position (from mini-STRIKE) as a function of the electrically measured ion current density, during a conditioning day.

to the change of complex Cs fluxes there is no longterm dependence [33]. Since the source performance depends on the amount of Cs present on the plasma grid, it is reasonable to assume that on a short timescale a correlation exists between the Cs density given by laser absorption and the Cs coverage on the PG; such dependence is lost when the source becomes well-cesiated.

The second interesting feature which emerges in a typical conditioning day at $\mathrm{z}=3 \mathrm{UP}$ is the quite different behavior of the five BES lines-of-sight. Figure 13 (left) shows the divergence of each BES-telescope as a function of the electrically measured ion current density $j_{e x}$; the data refer to a recent Cs conditioning day in hydrogen (April 2015). If the beam current density is relatively low (below $9 \mathrm{~mA} / \mathrm{cm}^{2}$ ), an increase in the local current density results in a decrease of all the divergences: this implies that the beam is locally under-perveance along all the five lines-of-sight, the most common situation in BATMAN.

If we consider the parabolic relationship between divergence and normalized perveance, each BES telescope can be represented by a point located on the left branch of the parabola (Figure 13, right): the perveance is different from point to point because the beam current shows a vertical asymmetry (effect (1) previously discussed). During the conditioning the increase of local current density leads to an increase of the local beam perveance for all the telescopes: the five points move along the parabola, from left to right, until the point most on the right, namely the BEStelescope with highest intensity, passes to the overperveance branch. This transition is schematically shown in Figure 13 (right). The first point to become over-perveance corresponds to BES-telescope \#5: when $j_{e x} \sim 9 \mathrm{~mA} / \mathrm{cm}^{2}$ the upper part of the beam

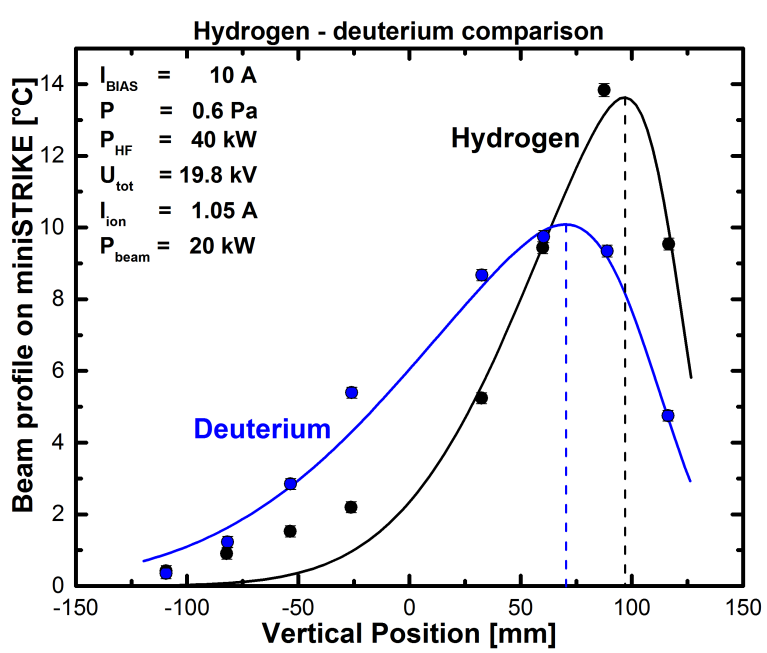

Figure 15. Beam vertical profiles from mini-STRIKE for a hydrogen and a deuterium beam: the source settings and the main beam parameters, indicated in the inlet, are the same for the two shots.

alone undergoes a under-perveance to over-perveance transition, while the rest of the beam remains underperveance. After the transition, the optical properties of the beam are no more uniform: further increase in the ion current density results in a de-focalization of the upper part of the beam (over-perveance) and in a focalization of the central and lower parts.

Such variations of the local optical properties of the beam affect the shape of the beam vertical profile: the beam center - namely the location of maximum heat flux - is expected to leave the regions which are de-focusing (over-perveance) and to move towards the ones which are focusing (under-perveance). This explains why the beam profile as given by miniSTRIKE shifts downwards after the under-perveance to over-perveance transition of the beam portion seen by BES 5 (Figure 14).

To sum up, again a good agreement exists between spectroscopic and calorimetric diagnostics: beam asymmetry factor $\nu$ and local beam divergence from spectroscopy - together with the vertical position of the beam - from calorimetry - evolve during a conditioning day at $\mathrm{z}=3 \mathrm{UP}$ in hydrogen, suggesting an increase of the top-bottom asymmetry of the Cs coverage at the plasma grid.

\subsection{Deuterium}

BATMAN source can be operated also in deuterium. The most evident feature of a $D^{-}$beam, with respect to a standard $H^{-}$beam, is a vertical profile broader and differently displaced (Figure 15). This is most probably a mass effect: the higher mass of deuterium results into a larger Larmor radius of the beam ions $\left(r_{L} \propto m\right)$, leading to a lower 
$v \times B$ deflection from the horizontal filter field, and into a higher normalized perveance $\left(\Pi / \Pi_{0} \propto \sqrt{m}\right)$, leading to a higher divergence of the (overlapping) beamlets which compose the beam. Furthermore, experimental measurements of the plasma density in front of the PG have shown that a deuterium plasma is more homogeneous that a hydrogen plasma [34]: this contributes to the shape of the beam vertical profile.

Similar conclusions can be drawn by looking at the beam profiles provided by mini-STRIKE, at the spectroscopic beam profiles given by the BES (as defined in section 3.2) or at the asymmetry factor $\nu$.

\section{Conclusion}

The main beam features of the BATMAN prototype source for the ITER neutral beam injection system - namely divergence, homogeneity and top-bottom asymmetries - have been investigated in different experimental scenarios by means of three diagnostic techniques, benchmarked against each other: Beam Emission Spectroscopy, the calorimeter mini-STRIKE and a copper calorimeter.

Clear correlations of the beam properties with the strength of the magnetic filter field and with the source conditioning have been found. The stronger the magnetic filter field in the beam region, the higher the top-bottom asymmetry, most likely a $v \times B$ effect. Moreover, operating the source at $\mathrm{z}=3 \mathrm{UP}$, all beam diagnostics show an increase of beam topbottom asymmetry during the conditioning; this evidence together with measurements of the density of neutral Cs in front of the plasma grid suggest an inhomogeneous $H^{-}$production at the plasma grid, presumably provoked by drifts in the plasma and an inhomogeneous Cs-coverage of the grid.

Finally, operation with $H^{-}$and $D^{-}$beams have been compared at fixed source settings (indicated in Figure 15); the $D^{-}$beam was found to be broader and vertically displaced with respect to a standard $H^{-}$ beam, probably the combined result of mass effects and a more homogeneous plasma facing the extraction system.

The mini-STRIKE calorimeter works well to obtain beam vertical profiles and power loads: vertical movements of the beam detected by mini-STRIKE agree with changes in the top-bottom beam asymmetry factor from spectroscopy (BES), although different distances of the two diagnostics from the last grid.

Beam emission spectroscopy emerged as a powerful diagnostic tool at least for two reasons. Firstly, the choice of the fitting function for the Doppler-shifted peak in a BES spectrum is not so critical since it does not affect the estimated beam divergence and intensity; an effect of the fitting function may appear for beams with much smaller divergence, a possibility that can be checked for instance in the SPIDER beam. Secondly, by using the asymmetry factor - defined as a proper combination of the BES intensities - it is possible to characterize in great detail the beam behavior and to draw the same conclusions obtained with calorimetry.

The negative ion beam for ITER NBI will be studied with BES both at SPIDER and MITICA; the work described in the paper provides a first insight into how a negative ion beam can be characterized with both spectroscopic and calorimetric measurements and can be considered a reference for similar future investigations in SPIDER. Also, the results presented indicate that a sufficient number of BES telescopes will be able to characterize well the negative ion beam for ITER NBI, in particular it will be possible to detect and investigate vertical movements of the beam, seen as changes in the asymmetry factor, and variations of the local beam divergence.

\section{Acknowledgments}

Useful discussion with L. Schiesko and D. Wünderlich and with all members of ITED group at IPP are truly acknowledged; the collaboration and help of S. Cristofaro, R. Delogu, A. Pimazzoni and R. Pasqualotto were highly appreciated.

\section{References}

[1] Hemsworth R et al. 2008 Review Scientific Instruments 79 doi: $10.1063 / 1.2814248$

[2] Speth E et al. 2006 Nuclear Fusion 46(6) 220-238 doi: 10.1088/0029-5515/46/6/S03

[3] Franzen P et al. 2015 Nuclear Fusion 55(5) 053005 doi: 10.1088/0029-5515/55/5/053005

[4] Cavenago M et al. 2014 Review of Scientific Instruments 85 doi: 10.1063/1.4824809

[5] Zacks J et al. 2013 AIP Conference Proceedings 1515 doi: $10.1063 / 1.4792829$

[6] Franzen P et al. 2011 Plasma Physics and Controlled Fusion 53 doi: 10.1088/0741-3335/53/11/115006

[7] Bacal M and Wada M 2015 Applied Physics Reviews 2 doi: 10.1063/1.4921298

[8] Belchenko $\mathrm{Y}$ et al. 1974 Nuclear Fusion 14(1) doi: 10.1088/0029-5515/14/1/017

[9] Froeschle $\mathrm{M}$ et al. 2009 Fusion Engineering and Design 84 788-792 doi: 10.1016/j.fusengdes.2008.12.063

[10] Fantz U et al. 2006 Nuclear Fusion 46(6) 297-306 doi: $10.1088 / 0029-5515 / 46 / 6 / \mathrm{S} 10$

[11] Zaniol B et al. 2012 Review of Scientific Instruments 83 doi: 10.1063/1.4705739

[12] Barbisan M et al. 2014 Review of Scientific Instruments 85 doi: 10.1063/1.4826325

[13] Geddes J et al. 1981 Journal of Physics B, Atomic and Molecular Physics 14 4837-4846 doi: 10.1088/00223700/14/24/018

[14] Barnett C et al. (ed) 1990 Atomic Data for Fusion. vol 1: Collisions of $\mathrm{H}, \mathrm{H}_{2}, \mathrm{He}$, and $\mathrm{Li}$ atoms and ions with atoms and molecule (ORNL-6086)

[15] Franzen P et al. 2011 AIP Conference Proceedings 1390 doi: $10.1063 / 1.3637401$ 
[16] Zaniol B 2011 Journal of Quantitative Spectroscopy and Radiative Transfer $112 \quad 513-518$ doi: 10.1016/j.jqsrt.2010.10.003

[17] Sonato P et al. 2009 Fusion Engineering and Design 84 269-274 doi: 10.1016/j.fusengdes.2008.11.095

[18] Serianni G et al. 2014 Review of Scientific Instruments 85 doi: 10.1063/1.4861391

[19] Serianni G et al. 2015 AIP Conference Proceedings 1655 doi: 10.1063/1.4916476

[20] Antoni V et al. 2015 AIP Conference Proceedings 1655 doi: $10.1063 / 1.4916474$

[21] Riedl R 2016 IPP, Private communication

[22] Franzen $\mathrm{P}$ et al. 2011 Nuclear Fusion 51(7) 073035 doi: 10.1088/0029-5515/51/7/073035

[23] Fantz U et al. 2009 Nuclear Fusion 49125007 doi:10.1088/0029-5515/49/12/125007

[24] Bonomo F and Schiesko L 2015 IPP, Private communication

[25] Humphries S 1990 Charged Particle Beams (Department of Electrical and Computer Engineering, University of New Mexico) chap 7

[26] Child C 1911 Physical Review (Series I) 32 492-511 doi: 10.1103/PhysRevSeriesI.32.492

[27] Barbisan M 2015 Beam emission spectroscopy studies in a $H^{-} / D^{-}$beam injector Ph.D. thesis University of Padua and Instituto Superior Técnico of Lisboa full text

[28] Fantz U et al. 2014 Plasma Sources Science and Technology 23 doi: 10.1088/0963-0252/23/4/044002

[29] Schiesko L et al. 2012 Plasma Physics and Controlled Fusion 54 doi: 10.1088/0741-3335/54/10/105002

[30] Schiesko L et al. 2012 Plasma Sources Science and Technology 21 doi: 10.1088/0963-0252/21/6/065007

[31] Gutser R et al. 2009 AIP Conference Proceedings 1097 doi: $10.1063 / 1.3112525$

[32] Wimmer C 2015 IPP, Private communication

[33] Wimmer C et al. 2013 AIP Conference Proceedings 1515 doi: 10.1063/1.4792791

[34] Fantz U et al. 2013 AIP Conference Proceedings 1515 doi: $10.1063 / 1.4792785$ 